\title{
Rapid Identification of Signature Moments to Improve Engagement in Population Health Programs
}

\author{
Timothy S. Wells, PhD, ${ }^{1}$ Kevin Hawkins, PhD, ${ }^{1}$ Gandhi R. Bhattarai, PhD, and Ellen R. Wicker, MHA ${ }^{2}$
}

\section{Background}

$\mathbf{P}$ OPULATION HEALTH PROGRAMS help people live healthier lives by addressing the multiple determinants of health, including the adequacy of medical care, public health interventions, challenges posed by the social and physical environment, genetic susceptibilities, and behavioral risk factors. ${ }^{1}$ Population health implies a focus on health care quality, with an emphasis on getting the right care to the right patient at the right time. ${ }^{2}$ Ideally, patients would self-identify (eg, "raise their hands") when they need help, but that often is not the case. This article discusses how important personal events, which we refer to as "signature moments," influence an individual's health journey. Then, we describe several techniques to identify these individuals in near real time after a signature moment, which may lead to better engagement in population health programs. Although there is growing interest in big data, we find value in the subset containing near real-time information to identify individuals who may benefit from participation in population health programs.

\section{Health Transitions and Signature Moments}

Health declines as we age, often marked by nodal events, such as a first heart attack or other impactful change. Transitions in health may be insidious or sudden. Examples with a slow onset include most cancers, the development of diabetes, or a number of other chronic conditions. In contrast, other health events have a sudden onset, such as a heart attack or fall resulting in a hip fracture. Even though some events are insidious, their diagnosis represents a change in health at a specific time, with each new diagnosis perhaps representing a life-altering change in the individual's health.

Diagnoses that negatively impact long-term health are significant milestones in an individual's health journey, and have been called "signature moments." These signature moments often motivate the individual to make significant changes in behavior: for example, with regard to smoking, alcohol, diet, exercise, and medication compliance. Some individuals try to modify their behavior alone, but most need assistance to succeed and, therefore, may be more motivated to participate in population health programs designed to help them live healthier lives. Identifying signature moments in near real time provides a means to engage these individuals at a time in their health journey when they may be more motivated to engage in a population health program.

\section{Engagement in Population Health Programs}

As with any health intervention initiative, engagement is critical to the success of population health programs. It seems logical to assume that population health programs, often provided at no additional cost to most employees in large companies and those covered by Medicare Advantage plans, would have high engagement rates. Yet, many programs find it challenging to engage individuals, with nonengagement rates at $70 \%-80 \% .^{3}$ Past research on engagement in care coordination programs indicates that individuals who perceived a benefit from the program, found the program convenient, needed a sounding board, or felt that they were not getting enough support from their medical providers were more likely to engage. ${ }^{4}$ Conversely, the top reasons for not engaging included failure to see added benefit from engagement and comfort with current health care processes. ${ }^{4}$

Most population health programs use an outbound model to contact potential participants. Specifically, health risk assessments and medical claims data are mined to identify either high-cost patients, patients with gaps in clinical care, or modifiable risks. Once identified, the claims data often need to be linked to administrative data to gather personal identifiers and contact information. The data may undergo additional cleaning and then are queued for an outbound contact. The outbound contact generally consists of a telephone call during which a trained nurse or engagement specialist talks with the individual and offers an invitation to participate in the program. Unfortunately, there typically is a significant lag

\footnotetext{
${ }^{1}$ Advanced Analytics, Optum, Ann Arbor, Michigan.

${ }^{2}$ Medicare Supplement Program, AARP Services, Inc., Washington, District of Columbia.
}

(C) Wells et al, 2017; Published by Mary Ann Liebert, Inc. This Open Access article is distributed under the terms of the Creative Commons Attribution Noncommercial License (http://creativecommons.org/licenses/by-nc/4.0/) which permits any noncommercial use, distribution, and reproduction in any medium, provided the original author(s) and the source are credited. 
between the patient's health care visit and the outbound call for a combination of reasons. First, it may take several months for a medical visit to appear in the claims data. Next, it can take 1 or more months for the claims data to be mined, individuals identified, and the data cleaned and queued. Finally, depending on the workload of the outbound callers and the number of times it takes to reach the individual, this process may take a month or longer. Given that it may take about 6 months from the event to the outbound call, it is unlikely that individuals are being contacted when they are most motivated to participate or make a change.

\section{Signature Moments and Behavior Change}

To our knowledge, there is no standard list of signature moments, but the 43 events listed in the Holmes and Rahe social readjustment rating scale $^{5}$ may reasonably be construed as signature moments, as the scale has been validated as a predictor of future illness. ${ }^{6}$ This questionnaire includes events related to personal relationships, death of a friend or relative, severe illness, changes in finances, changes in personal behavior or social activities, work-related stress, and changes in housing or educational settings.

Whether signature moments cause individuals to take action to change course in their health journey is dependent on many factors, such as those described in the Health Belief Model. ${ }^{7}$ In this model, an individual determines his or her perception of threat for a particular disease condition based on the condition's severity and susceptibility, as well as internal or external motivational factors. Internal cues may include current symptoms, whereas external cues may include information in the media, a similar illness in an acquaintance, or advice from a friend, family member, or medical professional. Then, whether the individual acts depends on the perceived threat, benefits, and barriers for various courses of action. One such signature moment leading to change occurs when someone needs care at an emergency department, as explained by Boudreaux et al. ${ }^{8}$ This group has described a "Sentinel Event Effect" as a health behavior change caused by a specific event, experience, or consequence of a risky behavior. Examples provided include tobacco cessation after a cardiac-related health problem or reduced alcohol consumption after a motor vehicle crash in which the driver was intoxicated. ${ }^{8}$ In both examples, the risky behavior resulted in treatment at an emergency department, which was the sentinel event (ie, "wake-up call") that motivated a change in health behavior. In a similar manner, a diagnosis of diabetes or heart disease may be the motivation needed to lose weight. ${ }^{9}$

\section{Identifying Signature Moments}

Currently, about 4 million people have an AARP ${ }^{\circledR}$ Medicare Supplement insurance (ie, Medigap) plan insured by UnitedHealthcare Insurance Company (for New York residents, UnitedHealthcare Insurance Company of New York). These plans help to defray the out-of-pocket costs not covered by traditional fee-for-service Medicare. Several tools and services, included at no extra charge to these individuals, result in real-time data. These are described in the following paragraphs.

Many insureds call in to Optum Consumer Sales and Services (Optum CSS) or the Nurse HealthLine. Optum CSS is Optum's contact center specializing in customer relationship management. Every interaction with a caller is maximized to promote the right products and services at the right time in the individual's health journey, building customer loyalty and adding program value. The most frequent inquiries are about billing, claims, demographic changes, product information, and rate changes. Individuals with an AARP-branded Medicare Supplement plan often reach out to Optum CSS after a signature moment (eg, inquiries about covered services). It is likely that an important key to engagement is timing; when these individuals call in (ie, "raise their hands"), they need to be engaged in near real time. As such, it is of interest to investigate opportunities to have Optum360 agents identify these signature moments and either offer a population health program or transfer the individual to a nurse who will further explore services that may provide a benefit.

Since 2008, the Nurse HealthLine has been available to all individuals with an AARP-branded Medicare Supplement plan. The Nurse HealthLine is a 24-hour resource that provides 2 main services: triage and nontriage support. Triage calls are those during which the staff members suggest the most appropriate place to seek health care for callers with health problems. Nontriage callers receive information about their insurance benefits and assistance with other services, such as referrals to community resources and suggestions for high-quality health care providers. Typically, each Nurse HealthLine staff member provides information about 2 or 3 of these services on each call. Although program staff members directly engage individuals in their care through triage calls, many of the nontriage calls are likely signature moments. For example, when individuals call looking for specialists in their area, or want to know whether a certain treatment is covered, these are signature moments.

A number of surveys are conducted among those with AARP-branded Medicare Supplement plans, and are another promising channel to identify individuals at the right time. A common type is the health risk appraisal, which is used to survey those who are new to the plan. Another common type, similar to the Consumer Assessment of Healthcare Providers and Systems survey, is administered to a random sample of insureds, asking about experiences and satisfaction with health care. The responses provided during these surveys can be used to identify the right program for the individual, whereas simply responding to the survey can illustrate a readiness to change. For example, those who respond to surveys are twice as likely to engage in current programs compared with those who do not respond.

In addition, about half of individuals with an AARPbranded Medicare Supplement plan also have an AARP MedicareRx Part D plan to help pay for prescription medication expenses. These data are available in near real time, and are now being investigated to determine how an analysis of new prescriptions can be used to identify individuals who have signature moments associated with a change in their health journey. In addition, the first time a patient receives a drug for a chronic condition or a pain reliever, where the prescription is filled also is telling (eg, long-term care facility).

Technological advances also can help identify signature moments. For example, with biometric monitoring of congestive heart failure, patients are provided a scale that telephonically reports weight. Individuals are asked to weigh themselves on a daily basis, and these data can be monitored 
for changes in weight. In addition, program staff can contact individuals who fail to weigh-in to ensure they are not having difficulty with the equipment or a problem with their health. In several cases, individuals had not weighed-in because they were hospitalized, and absence of the daily weight reporting provided an early indicator of the hospitalization (ie, a signature moment). As other technologies (eg, smartphones, personal exercise monitors, and other wearables) become more popular, it may be possible to leverage these technologies to provide early identification of signature moments.

Finally, aging into Medicare (and/or retirement) is likely a signature moment and these individuals need assistance to understand the benefits available. To help with the transition into Medicare, older adults need assistance sorting out the dozens of options along with their associated costs and coverages. This is especially true because, in the past, these decisions often were made by an individual's employer plan or his or her spouse's employer plan. To provide these individuals with the information they need, many insurers provide a welcome packet that explains Medicare and the various services provided to help defray Medicare's out-of-pocket expenses. Identifying individuals new to Medicare is another opportunity to address signature moments in near real time.

\section{Conclusions}

Population health programs help individuals with high health care needs manage their health issues through an integrated team approach. Although proven beneficial, it has been challenging to encourage individuals to participate. Using innovative methods to identify individuals who have signature moments in near real time may improve participation rates. In this article, we have proposed the use of readily available data in new ways to drive member engagement. We also foresee that wearable technologies may be leveraged in the future. The ability to identify signature moments in near real time can increase participation in population health programs, and individuals who participate are more likely to have improved quality of care and reduced health care costs.

\section{Acknowledgment}

The authors thank Stephanie MacLeod, MS, for her editorial assistance and critical review of this article.

\section{Author Disclosure Statement}

Drs. Wells, Hawkins, and Bhattarai, and Ms. Wicker declared the following potential conflicts of interest with respect to the research, authorship, and/or publication of this article: This research work was funded by the Supplemental
Health Insurance Program. Drs. Hawkins, Wells, and Bhattarai are all employed by UnitedHealth Group and have stock with UnitedHealth Group. Ms. Wicker is employed by AARP Services, Inc. However, their compensation was not dependent upon the results obtained in this research, and the investigators retained full independence in the conduct of this research.

\section{References}

1. Kindig D, Stoddart G. What is population health? Am J Public Health 2003;93:380-383.

2. Clancy CM. Statement by Carolyn M. Clancy M.D. Director, Agency for Health Care Research and Quality, U.S. Department of Health and Human Services, on What is Health Care Quality and Who Decides? Before Committee on Finance Subcommittee on Health Care, United States Senate, Wednesday March 18, 2009. www.hhs.gov/asl/testify/2009/ 03/t20090318b.html Accessed September 27, 2014.

3. Chen A, Brown B, Archibald N, Aliotta S, Fox PD. Best Practices in Coordinated Care. 2000. www.cms.gov/Medicare/ Demonstration-Projects/DemoProjectsEvalRpts/downloads/cc_ full_report.pdf Accessed October 7, 2015.

4. Hawkins K, Wells TS, Hommer CE, Ozminkowski RJ, Richards DM, Yeh CS. Factors driving engagement decisions in care coordination programs. Prof Case Manag 2014;19: 216-223.

5. Masuda M, Holmes TH. The Social Readjustment Rating Scale: a cross-cultural study of Japanese and Americans. J Psychosom Res 1967;11:227-237.

6. Rahe RH, Mahan JL Jr., Arthur RJ. Prediction of near-future health change from subjects' preceding life changes. J Psychosom Res 1970;14:401-406.

7. Rosenstock IM. Why people use health services. Milbank Mem Fund Q 1966;44(3 Suppl):94-127.

8. Boudreaux ED, Bock B, O'Hea E. When an event sparks behavior change: an introduction to the sentinel event method of dynamic model building and its application to emergency medicine. Acad Emerg Med 2012;19:329-335.

9. Zelman KM. Tips from 'The Biggest Loser': an interview with Cherly Forberg, RD. 2009. www.webmd.com/diet/ obesity/expert-qa-tips-from-the-biggest-loser Accessed January $13,2016$.

Address correspondence to: Timothy S. Wells, PhD Advanced Analytics Optum

315 East Eisenhower Parkway Suite 305 Ann Arbor, MI 48108

E-mail: tim.wells@optum.com 